\title{
Initial ovarian sensitivity index predicts embryo quality and pregnancy potential in the first days of controlled ovarian stimulation
}

David Camargo-Mattos ${ }^{1}$, Uziel García', Felipe Camargo-Diaz' , Ginna Ortiz¹ , Ivan Madrazo ${ }^{1}$ and Esther Lopez-Bayghen ${ }^{2^{*}}$ (D)

\begin{abstract}
Background: To determine if a modified ovarian sensitivity index (MOSI), based on initial follicular measurements and the initial follicle-stimulating hormone (FSH) dose, can predict the production of high-quality embryos for successful implantation during in vitro fertilization (IVF).

Methods: This study consisted of two phases: 1) a retrospective study and 2) a prospective observational study. For the first phase, 363 patients charts were reviewed, of which 283 had embryos transferred. All women underwent a standardized antagonist-based IVF protocol. At the first follow-up (Day 3/4), the number and size of the follicles were determined. MOSI was calculated as In (number follicles ( $\geq 6 \mathrm{~mm}$ ) $\times 1000$ / FSH initial dose). Afterward, the number and quality of the ova, embryo development, and the number and quality of the blastocysts were determined. Embryo implantation was confirmed by $\beta$-hCG. For the second phase, 337 IVF cycles were followed to determine MOSI's accuracy.

Results: MOSI could predict the production of $\geq 4$ high-quality embryos by Day 2 ( $\mathrm{AUC}=0.69,95 \% \mathrm{Cl}: 0.63-0.75$ ), $\geq 2$ blastocysts ( $A \cup C=0.74,95 \% \mathrm{Cl}: 0.68-0.79$ ), and $\geq 35 \%$ rate of blastocyst formation ( $\mathrm{AUC}=0.65,95 \% \mathrm{Cl}: 0.58-0.72$ ). Using linear regression, MOSI was highly associated with the number of ova captured ( $\beta=5.15)$, MIl oocytes $(\beta=4.31)$, embryos produced $(\beta=2.90)$, high-quality embryos ( $\beta=0.98$ ), and the blastocyst formation rate $(\beta=0.06, p<0.01)$. Using logistic regression, MOSI was highly associated with achieving $\geq 4$ high-quality embryos (odds ratio $=2.80,95 \% \mathrm{Cl}: 1.90-4.13$ ), $\geq 2$ blastocysts (odds ratio $=3.40,95 \% \mathrm{Cl}$ : 2.33-4.95), and $\geq 35 \%$ blastocysts formation rate (odds ratio $=1.96,95 \% \mathrm{Cl}: 1.31-2.92)$. This effect was independent of age, BMI, and antral follicle count. For implantation, MOSI was significantly associated with successful implantation (odds ratio $=1.79$, 95\%(l:1.25-2.57). For the prospective study, MOSI was highly accurate at predicting $\geq 6$ high-quality embryos on Day 2 (accuracy $=68.5 \%), \geq 6$ blastocysts (accuracy $=68.0 \%$ ), and a blastocyst formation rate of $\geq 35 \%$ (accuracy $=61.4 \%$ ).

(Continued on next page)
\end{abstract}

\footnotetext{
* Correspondence: ebayghen@cinvestav.mx

${ }^{2}$ Departamento de Toxicología, Centro de Investigación y de Estudios Avanzados del Instituto Politécnico Nacional (CINVESTAV-IPN), Avenida Instituto Politécnico Nacional 2508, San Pedro Zacatenco, 07360 México City, Mexico

Full list of author information is available at the end of the article
}

(c) The Author(s). 2020 Open Access This article is licensed under a Creative Commons Attribution 4.0 International License, which permits use, sharing, adaptation, distribution and reproduction in any medium or format, as long as you give appropriate credit to the original author(s) and the source, provide a link to the Creative Commons licence, and indicate if changes were made. The images or other third party material in this article are included in the article's Creative Commons licence, unless indicated otherwise in a credit line to the material. If material is not included in the article's Creative Commons licence and your intended use is not permitted by statutory regulation or exceeds the permitted use, you will need to obtain permission directly from the copyright holder. To view a copy of this licence, visit http://creativecommons.org/licenses/by/4.0/ The Creative Commons Public Domain Dedication waiver (http://creativecommons.org/publicdomain/zero/1.0/) applies to the data made available in this article, unless otherwise stated in a credit line to the data. 
(Continued from previous page)

Conclusion: MOSI was highly correlated with key IVF parameters that are associated with achieved pregnancy. Using this index with antagonist cycles, clinicians may opt to stop an IVF cycle, under the assumption that the cycle will fail to produce good blastocysts, preventing wasting the patient's resources and time.

Keywords: Poor ovarian response, Ovarian sensitivity index, Antral follicle count, In vitro fertilization, Follicle-stimulating hormone

\section{Background}

Currently, one in six couples have at least one partner that suffers from infertility, the failure of achieving a viable gestation after a year of unprotected sex [1]. Over the past 40 years, assisted reproduction technologies (ART) have improved the pregnancy rates of infertile couples [2]. However, ART remains an inefficient process due to the multiple sources of variability, affecting the quantity and quality of the ova collected [2]. One of the leading causes for variability between ova cohorts is how patients respond to ovarian stimulation with follicle-stimulating hormone (FSH) or human menopausal gonadotropin (hMG) [3]. It is well documented that patients will have different responses to FSH and hMG, according to their ovarian reserve [4], age, weight, and presence of insulin resistance [3]. Depending upon the initial dose-response, the physician may adjust follow-up doses, but what remains is a lack of knowledge of the predictability of the initial dose.

One of the most critical factors for in vitro fertilization (IVF) success is to have a good follicular response, the production, and release of a sufficient number of ova (between 4 and 15) [5]. Numerous reports have demonstrated that the number of ova collected is associated with improved IVF outcomes and live birth rates [6]. Nevertheless, Rombauts et al. demonstrated that during sequential stimulation cycles, different responses could occur for each cycle to generate good embryos [7]. Therefore, a question remains, can a physician predict the potential yields of key IVF parameters, such as the number of ova collected, the number of high-quality embryos, and the number of blastocysts formed. The optimal time to answer this question would be the first measure of serum estradiol (E2) during the third or fourth day of stimulation.

The ovarian sensitivity index (OSI), developed by Biasoni et al. [8] and refined by Huber et al. [9], measures the ovarian response to the total FSH dose. However, using the total FSH dose may mislead the physician to believe that the ovarian stimulation was acceptable and too late to stop the cycle or change strategies to minimize losses. Therefore, we posit that using the initial ovarian stimulation measurements may be more accurate for determining the ovarian response. A modified OSI (MOSI) that uses the number of follicles detected on Day 3 or Day 4 during ovarian stimulation could be a better predictor. Follicles $\geq 6 \mathrm{~mm}$ on Day 3 or Day 4 of stimulation have been shown to have the highest potential to end up as mature follicles and are more likely to produce the best ovules [10]. Therefore, this research aims to determine the predictability of obtaining good quality ova that could lead to highquality embryos in patients undergoing IVF, using the number of follicles during Day 3 or Day 4 of ovarian stimulation with the initial FSH dose.

\section{Methods and materials Study population}

This study was performed in 2 phases: 1) a retrospective study that examined the association between MOSI and certain IVF parameters and 2) a prospective observational study to assess the effectiveness of MOSI. Since we are testing MOSI as a potential tool for IVF, we utilized a general IVF population, and patients would only be excluded for particular circumstances. For the retrospective study, a database with de-identified, non-coded data from women at the Instituto de Infertilidad y Genética SC, Ingenes, Mexico, was obtained (May 2018 to December 2018). Data were from women attending the clinic and underwent a standardized IVF protocol, using their own ova (no donor ova). Here, at our clinic, $80 \%$ of IVF cycles are performed with the antagonist protocol; therefore, only women that underwent an antagonist protocol were considered. There was no restriction on sperm source. The inclusion criteria were as followed: IVF cycles with controlled ovarian stimulation using the antagonist protocol; the patient's own ova were used; had between one and three embryos transferred, and a written and signed consent allowed us to use their data. Patients were excluded if during the previous 6 months, they had any hormonal treatment, were diabetic, had a high-risk of ovarian hyperstimulation syndrome, or if their IVF cycle resulted in an ectopic pregnancy. All patients with donated ova or incomplete data were also excluded. For the prospective study, another cohort was collected from January 2019 to January 2020 for testing the efficiency of MOSI. The same inclusion and exclusion criteria for the retrospective study were used for this study. This cohort consisted of 337 IVF cycles.

\section{Ovarian stimulation protocol}

Ovarian stimulations were performed by a group of 27 doctors under a unified protocol, reviewed by the 
Ingenes Quality Control Group of Medical Indicators (IQCG-IMED 2019). This internal entity reviews the Institute's performance for indicators of medical diagnoses, embryonic development, transfer results, and pregnancy rates to detect areas of opportunity and correct errors, based on the SART parameters [11].

All patients were subjected to a controlled ovarian stimulation for 10 days with gonadotropin-releasing hormone antagonists. For each patient, the ovarian reserve was determined by the antral follicle count (AFC) using ultrasound. Anti-Müllerian hormone (AMH) levels were not determined as we had noticed the high variability between lab results as well as the high costs the patients would endure. AFC was performed, and based on the patient's BMI, age, and size of the antral follicles, the initial FSH dose was determined, which remained unchanged for the first three to four days. Ovarian response was assessed measuring serum E2 levels, and follicular development was evaluated by ultrasound examination. To adjust the FSH dose, ultrasonographic follow-ups were programmed on alternate days according to patient availability, serum E2, and progesterone levels, and according to the Institute's clinical criteria. Ultrasound measurements were made as an average of two perpendicular measurements of each of the follicles observed, including the follicle wall.

Follicular puncture for oocyte collection was performed under general anesthesia at the end of hormonal stimulation (10-14 days). Oocyte retrieval was conducted $36 \mathrm{~h}$ after ovulation was induced with human chorionic gonadotropin (hCG; 10,000 IU Choragon, Ferring Pharmaceuticals, or $6500 \mathrm{IU}$ Ovidrel, Merck Laboratories). Transvaginal ultrasound guidance was used to locate mature follicles, and 3-5 $\mathrm{ml}$ of follicular fluid containing the oocytes were extracted using a specialized suction system. Samples were analyzed using a stereoscopic microscope in order to locate the oocytes, which were kept at $37.5^{\circ} \mathrm{C}$ in an atmosphere of $8.3 \% \mathrm{CO}_{2}$ until fertilization. An embryologist monitored and recorded the information about fertilization, embryo development, embryo morphology, transfer, and pregnancy for each oocyte. The number and quality of the retrieved oocytes were assessed using their morphological parameters (granulosa expansion, oocyte maturity (MI, MII, and VG), quality of the cytoplasm, zona pellucida, and polar body).

Embryo quality was assessed on Day 2 of embryonic development for indicators of high-quality embryos (symmetrical, consisting of 4 cells, and $<10 \%$ fragmentation). Embryonic morphological parameters evaluated were weighed into a matrix to rate each oocyte-embryo, with the sum of the values rated on a scale of 0 (low quality) to 12 (high quality). Selection for embryo transfer was made on Day 3 or Day 5 of development according to the morphological assessment, using the criteria established by the Istanbul Consensus Workshop on Embryo Assessment [12]. Our protocol defines that with $<4$ good cleavage-stage embryos, the transfer is performed on Day 3. The highest quality embryos were transferred. $\beta$-hCG values confirmed clinical pregnancy $(>10 \mathrm{mUI} / \mathrm{ml}$ on Day 14). The number of embryos transferred was determined by the number of high-quality embryos achieving full development, patient results from previous attempts, and the opinion of the clinician.

\section{IVF outcome measurements}

An embryologist monitored and recorded all information about ovarian volume, AFC, the number of ova captured and MII ova, serum E2 levels, embryonic quality (determined by the number of high-quality embryos on Day 2 of embryonic development) and their rate (compared to the total number of ova captured), as well as the number embryos able to reach a blastocyst stage on Day 5 of embryonic development and the rate of ovules that generated blastocysts. AFC was defined as the total number of follicles measuring between 2 and $10 \mathrm{~mm}$ in diameter that were observed during the early follicular phase by transvaginal ultrasound.

\section{OSI and MOSI}

OSI was calculated as the total oocytes captured divided by the total FSH dose multiplied by 1000 , whereas the MOSI was calculated as the total number of follicles on Day 3 or Day 4 ( $\geq 6 \mathrm{~mm}$ ) divided by the initial dose of FSH multiplied by 1000 . Due to the presence of a skewed distribution, both indices were ln-transformed. Using the method described in Huber et al., the cutoff levels for poor, normal, and high responders were determined [9]. Patients that were classified as a potential poor or high responders were explained the potential outcomes associated with the classification by the physician. Afterward, the decision to continue with the cycle was given by the patient without severe objection by the physician.

\section{Sample size estimation}

At Ingenes, during 2018, 1447 IVF cycles were performed. Assuming a standard deviation of 31.98 and using a $95 \%$ confidence level with a maximum accepted error of $5 \%$, the optimal sample size of 304 cycles was calculated using the PS Power and Sample Size Calculations software for MS Windows, version 3.0.11 [13].

\section{Statistics}

The primary outcome was the association between MOSI and the number of blastocysts and the number of high-quality embryos, whereas the secondary outcomes were the number of ova captured and the number of MII ova. All analyses were performed using Statistical Package for the Social Sciences version 26 (SPSS, IBM 
Corp., Armonk, NY, USA). Data are presented as either mean \pm standard deviation, frequencies, or percentages. The normality of continuous data was determined using the Shapiro-Wilk test. To graphically assess the association between MOSI and the IVF parameters, we used the Generalized Additive Model to plot the variables, whereas, due to the shape of the plot, linear regression was used to quantify the association. Multivariate logistic regression was used to determine the predictability of MOSI for the IVF parameters, whereas Receiver Operator Characteristic (ROC) curves were used to compare the predictability between OSI and MOSI for IVF parameters using the Hanley and McNeil method [14]. ROCs were used to determining possible cutoffs using the Youden Index (Sensitivity + Specificity -1$)$. Cohen's kappa (к) was calculated to determine the inter-assay agreement. A $p$-value $<$ 0.05 (two-tailed) was considered significant.

\section{Results}

\section{Characteristics of the study population}

During the collection time, data from 947 patients (1447 cycles) were examined, of which $44.8 \%$ of these cycles were performed with donated ova and were excluded. 523 patients were identified as having IVF cycles with their own ova. One hundred sixty patients were eliminated because their data were incomplete, leaving 363 patients for this study. Only 283 patients had between one and three embryos transferred, which means that 80 cycles failed to produce a viable embryo (blocking), had aneuploid embryos, had the embryos frozen, or the patient elected not to continue with IVF. The cohort characteristics are summarized in Table 1. The main reasons for IVF were a low response, advanced age, or PCOS. The distribution of MOSI was non-parametric and was $\ln$ transformed (Fig. 1). When classifying the patients as poor, normal, or high responders, there was a $78 \%$ agreement between the OSI and MOSI (a moderate agreement, $\mathrm{K}=0.447, p<0.001$, data not shown).

\section{OSI and MOSI predict IVF parameters in infertile women from Central Mexico}

We compared the efficiency of OSI and MOSI to predict IVF parameters using ROCs. For producing $\geq 4$ high-quality embryos by Day 2, OSI was superior to MOSI (OSI: AUC = 0.79 , 95\%CI: $0.75-0.84, p<0.001$ versus MOSI: $\mathrm{AUC}=$ 0.69, 95\%CI: 0.63-0.75, $p<0.001$, pcomparison $<0.001$, Fig. 2a). Interestingly, neither index could predict $\mathrm{a} \geq 35 \%$ rate of high-quality embryos per the total number of ova captured (OSI: $\mathrm{AUC}=0.47, \quad 95 \% \mathrm{CI}: 0.40-0.54, p=0.407$ versus MOSI: $\mathrm{AUC}=0.50,95 \% \mathrm{CI}: 0.43-0.58, p=0.964, \mathrm{p}_{\text {compari- }}$ son $=0.239$, Fig. $2 \mathrm{~b}$ ). For producing $\geq 2$ blastocysts, OSI was again superior to MOSI (OSI: $A U C=0.82$, 95\%CI: $0.77-0.86, p<0.001$ versus MOSI: AUC = 0.74, 95\%CI: $0.68-0.79, p<0.001, \mathrm{p}_{\text {comparison }}<0.001$,
Table 1 Patient characteristics

\begin{tabular}{|c|c|}
\hline Variable & Value $^{a}$ \\
\hline Sample size (n) & 363 \\
\hline Age (years) & $36.1 \pm 4.6(19-45)$ \\
\hline BMI $\left(\mathrm{kg} / \mathrm{m}^{2}\right)$ & $25.9 \pm 4.3(18.8-38.9)$ \\
\hline \multicolumn{2}{|l|}{ Infertility factor $(n, \%)$} \\
\hline None indicated/normal & $33,9.1 \%$ \\
\hline Anovulation & $8,2.2 \%$ \\
\hline Low response & $80,22.0 \%$ \\
\hline Advanced age & $83,22.9 \%$ \\
\hline Endometriosis & $34,9.4 \%$ \\
\hline PCOS & $73,20.1 \%$ \\
\hline Tubal factor & $35,9.6 \%$ \\
\hline Other & $17,4.7 \%$ \\
\hline \multicolumn{2}{|l|}{ FSH (IU/L) } \\
\hline Initial & $303 \pm 78(75-450)$ \\
\hline Total & $3104 \pm 992(75-6000)$ \\
\hline Antral follicle count (n) & $12.0 \pm 8.3(0-46)$ \\
\hline \multicolumn{2}{|l|}{ Day 3-4 follicle count (n) } \\
\hline$<6 \mathrm{~mm}$ & $3.2 \pm 3.9(0-40)$ \\
\hline 6 to $8 \mathrm{~mm}$ & $5.1 \pm 3.9(0-22)$ \\
\hline$>8 \mathrm{~mm}$ & $5.1 \pm 4.2(0-26)$ \\
\hline \multicolumn{2}{|l|}{ OSI-modified (MOSI) } \\
\hline Score & $38.6 \pm 28.5(2.2-193.3)$ \\
\hline Ln transformed & $3.4 \pm 0.8(0.8-5.3)$ \\
\hline Ova captured (n) & $12.7 \pm 8.0(1-63)$ \\
\hline Mll ova (n) & $10.6 \pm 7.0(0-39)$ \\
\hline MII/Ova captured (\%) & $82.7 \pm 17.0(0.0-29.0)$ \\
\hline \multicolumn{2}{|l|}{ Ovarian sensitivity index (OSI) } \\
\hline Score & $4.7 \pm 3.7(0.2-22.7)$ \\
\hline Ln transformed & $1.2 \pm 0.9(-1.6-3.1)$ \\
\hline Total embryos on Day 2 (n) & $7.1 \pm 5.3(0-29)$ \\
\hline High-quality embryos (n) & $2.6 \pm 2.7(0-15)$ \\
\hline High-quality embryos/Ova captured (\%) & $21.5 \pm 19.3(0-100)$ \\
\hline Blastocyst (n) & $2.5 \pm 3.2(0-16)$ \\
\hline Blastocyst /Ova captured (\%) & $16.1 \pm 18.7(0-100)$ \\
\hline
\end{tabular}

Values, unless indicated otherwise, are mean \pm standard deviation (minimum-maximum)

Fig. 2c). However, both indices could predict $a \geq 35 \%$ rate of blastocyst formation per the total number of ova captured and neither indices were superior (OSI: AUC $=0.69, \quad 95 \% \mathrm{CI}: \quad 0.63-0.75, \quad p<0.001 \quad$ versus MOSI: $\mathrm{AUC}=0.65,95 \% \mathrm{CI}: 0.58-0.72, p<0.001, \mathrm{p}_{\text {com }}$ parison $=0.244$, Fig. 2d). These results suggest that OSI and MOSI can predict certain IVF parameters, but neither index is better at predicting the blastocyst formation rate. 
A

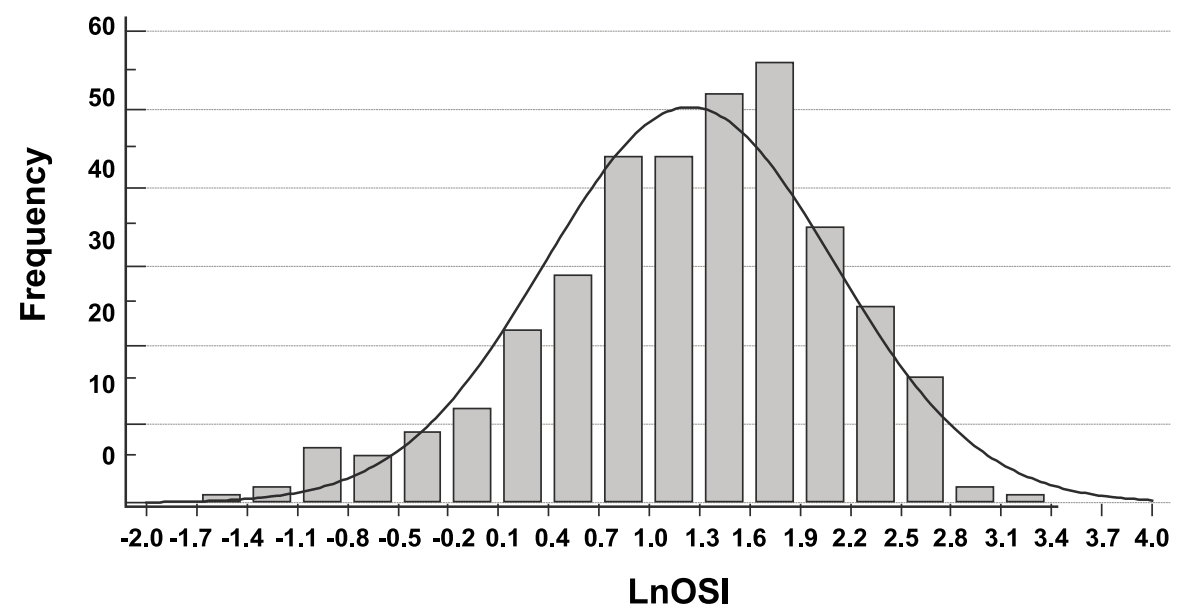

B

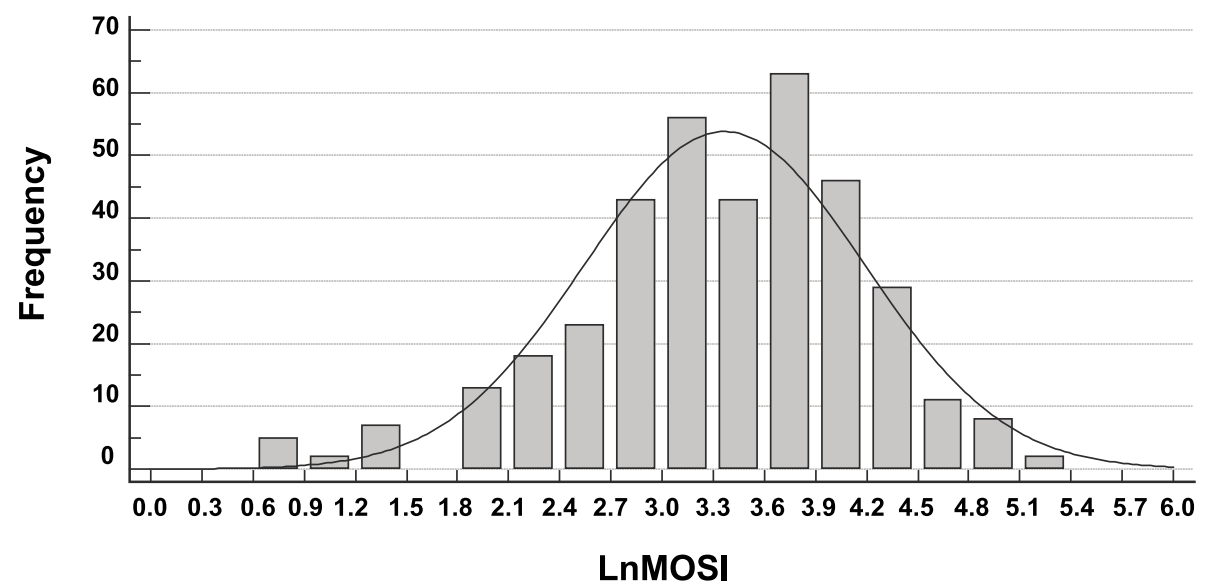

Fig. 1 Ln-transformed distribution of (a) ovarian sensitivity index (OSI) and (b) the modified OSI (MOSI) of the study population

MOSI predicts high-quality ovules and embryos

Graphic representation of MOSI and the total number of ova captured (Fig. 3a), MII oocytes (Fig. 3b), highquality embryos (Fig. 3c), and blastocysts (Fig. 3d) demonstrate a positive correlation between MOSI and the IVF parameters. The strength of the association between MOSI and the IVF parameters was measured by linear regression (Table 2). MOSI was highly associated with the number of ova captured (5.15 ova per a MOSI unit), the number of MII oocytes (4.31 MII oocytes per a MOSI unit), the number of total embryos produced (2.90 embryos per a MOSI unit), the number of high-quality embryos (0.98 embryos per a MOSI unit), the number of the blastocyst (1.43 embryos per a MOSI unit), and the blastocyst formation rate (6\% per a MOSI unit).

Using logistic regression, we determined the ability to achieve key goals associated with better IVF outcomes (Table 3). MOSI was highly associated with achieving $\geq 4$ high-quality embryos and $\geq 2$ blastocysts, and this effect was independent of age and BMI (model 1) and AFC (model 2). Moreover, achieving a rate of $\geq 35 \%$ blastocysts per the total number of ova captured were also significantly associated with MOSI and independent of age, BMI, and AFC. For embryo implantation, MOSI was significantly associated with implantation; however, this effect was lost when AFC was considered. Lastly, another cohort was collected 1 year later. Using the ROCs (Fig. 2), cutoffs were calculated using the highest Youden Index score. These cutoff values were used to assess the test accuracy. For each parameter, MOSI resulted in at least $60 \%$ accuracy (Table 4). Moreover, the Negative Predictive Value (NPV) was higher than $80 \%$ for each parameter.

\section{Discussion}

Ovarian reserves are shown to decrease with a woman's age $[4,15]$, but women of the same age can respond 
A

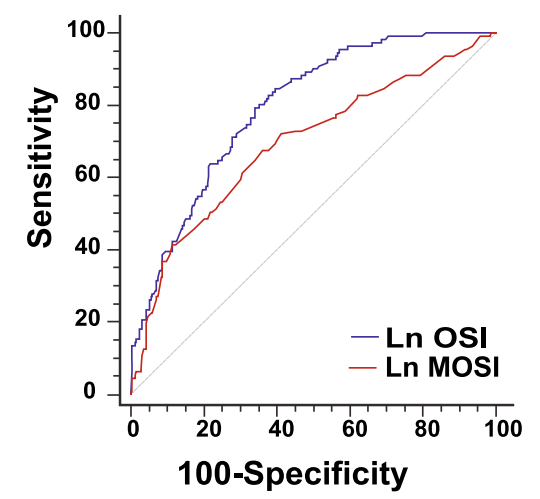

C

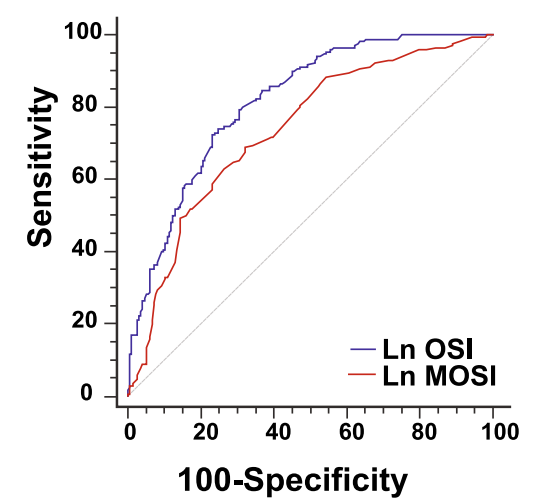

B

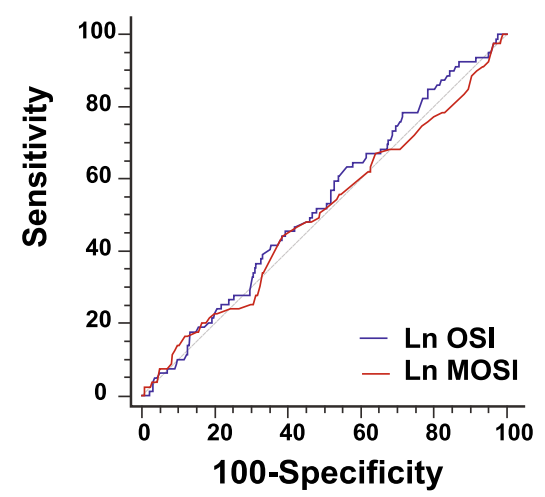

D

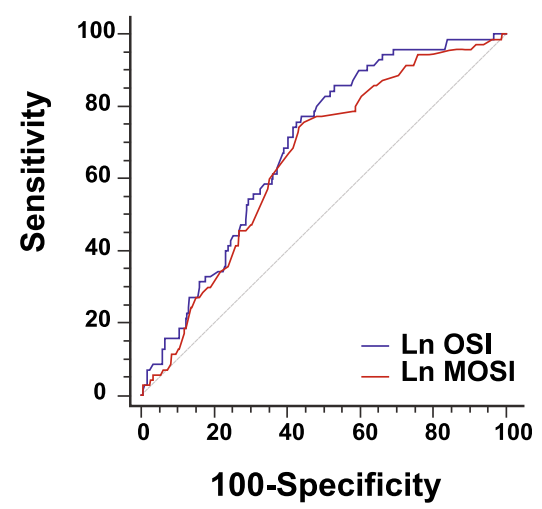

Fig. 2 The predictability of ovarian sensitivity index (OSI) and modified ovarian sensitivity index (MOSI) for in vitro fertilization outcomes. Receiver operating characteristic curves were constructed to determine if OSI (blue line) or the MOSI (red line) can predict $\mathbf{a} \geq 4$ high-quality embryos, $\mathbf{b}$ a rate $\geq 35 \%$ for the number of high-quality embryos/the number of ova captured, $\mathbf{c} \geq 2$ blastocyst, and $\mathbf{d}$ a rate $\geq 35 \%$ for the number of blastocysts/the number of ova captured. Comparison of Area Under Curve between predictors was performed using SPSS v26

differently to FSH stimulation [10, 15]. When a patient is undergoing IVF, the first time point in which the ovarian response can be measured is between three to 4 days after the initial FSH dose. The question remains, can this initial response determine the feasibility of the IVF cycle to produce viable embryos for implantation? Indeed, the response on Day 3 or 4 after stimulation did highly associate with ova production and of the number of embryos that developed.

An inadequate response to $\mathrm{FSH}$ has been correlated with diminished ova production, which significantly affects the possibility of an ideal gestation in ART [16]. It is known that in high-response cycles when there are too many follicles ( $>25$ ova), the number of genetic alterations is augmented [17, 18]. When $<6$ ova are obtained, there are less high-quality ova, and the IVF cycle is more likely to produced sub-optimal embryos [4]. Here, MOSI did correlate with the number of ova isolated as well as the MII oocytes count (Fig. 3). Therefore, this index does present with the ability to identify IVF cycles in which the cycle may fail to produce a sufficient number of ova or an excessive amount. Thus, MOSI could aid physicians in deciding to continue a cycle.

Huber et al. described the capabilities of OSI, in which even the possibility of pregnancy was predicted, but with the inconvenience of utilizing this index only after the ova were collected and the ovarian stimulation-protocol was completed [9]. This has an unfortunate consequence, given the fact that the main costs of an IVF cycle occur once the ova are collected. If the IVF cycle results in poor-quality ova, there is no possibility of stopping the procedure in time to restart the cycle to achieve a better stimulation [19]. Hassan et al. described the follicular sensitivity index (FSI). Although this model predicts the possibility of achieving a pregnancy, it is necessary to have finished the ovarian stimulation, as it uses total FSH dose. The FSI cannot predict from the beginning of the stimulation if high-quality embryos will be obtained [20]. Again, the clinician would try to achieve clinical pregnancy with embryos whose quality, in some cases, is not sufficient. Here, MOSI could determine, with similar efficiency as OSI and FSI, if clinical 
A

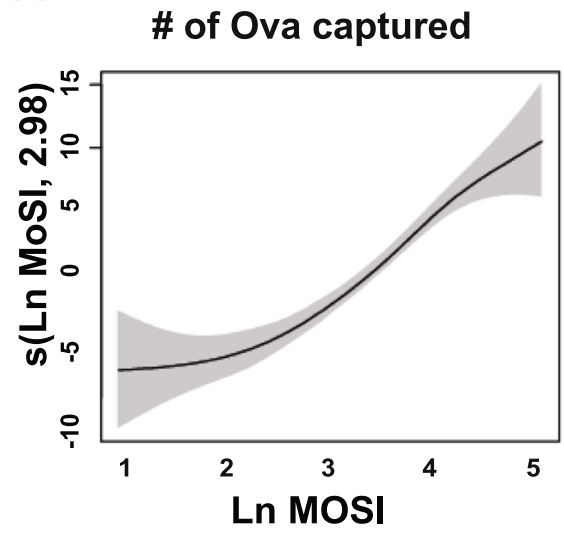

C
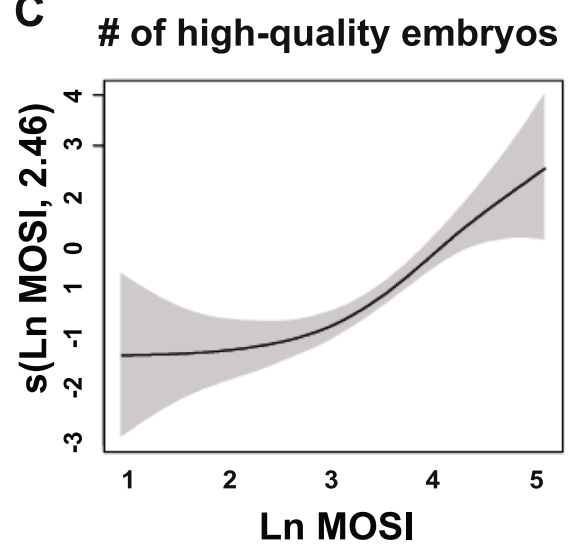

B

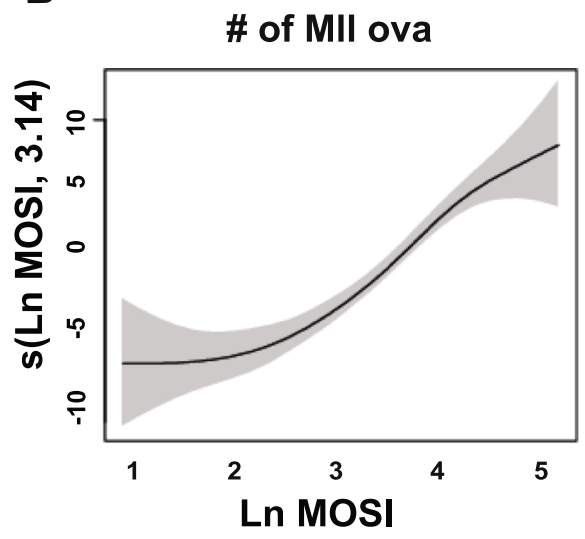

D \# of blastocyst (Day 5)

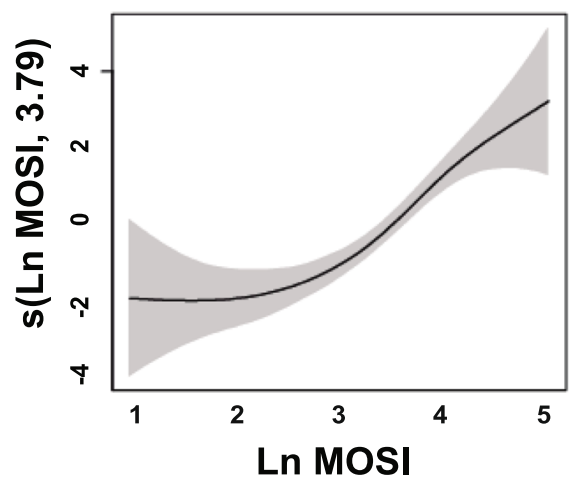

Fig. 3 Fitted values for a number of ova captured, b the number of Mll ova, c number of high-quality embryos, and $\mathbf{d}$ number of blastocysts on Day 5 according to modified ovarian sensitivity index (MOSI) using Generalized additive model with smoothing splines

pregnancy was achieved, but MOSI is doing it at an early phase.

Currently, depending on the IVF laboratory, it has been determined that only between 24 and $50 \%$ of the ova obtained will develop into a viable blastocyst that can be transferred [21]. Another factor for having viable blastocysts for transfer is if there are at least 4 highquality embryos on Day 2, which is correlated with ova capture. This has led to most fertility facilities to try to obtain at least 6 ova during the harvesting step. Numerous studies have examined how the FSH dose affects the follicles observed by ultrasound and the synchrony in their growth $[10,22,23]$. The changes in serum E2 and progesterone levels as well as ultrasound measurements of folliculometry [24] can serve as tools to predict the correct evolution of the stimulation; nevertheless, they remain insufficient. MOSI highly correlated with ova captured and with having at least 4 high-quality embryos

Table 2 Linear regression results

\begin{tabular}{|c|c|c|c|c|}
\hline Category & Correlation coefficient & Beta & Standard Error & $P$-value \\
\hline Ova captured & 0.510 & 5.15 & 0.46 & $<0.001$ \\
\hline Mll ova & 0.493 & 4.31 & 0.40 & $<0.001$ \\
\hline Mll ova/ Ova capture rate & 0.001 & 0.00 & 0.01 & 0.979 \\
\hline Embryos & 0.445 & 2.90 & 0.31 & $<0.001$ \\
\hline High-quality embryos & 0.287 & 0.98 & 0.17 & $<0.001$ \\
\hline High-quality embryos/Ova capture rate & 0.014 & 0.00 & 0.01 & 0.795 \\
\hline Blastocyst & 0.356 & 1.43 & 0.20 & $<0.001$ \\
\hline Blastocyst/ Ova capture rate & 0.265 & 0.06 & 0.01 & $<0.001$ \\
\hline
\end{tabular}


Table 3 Logistic regression

\begin{tabular}{|c|c|c|c|}
\hline Category & Crude model & Model $1^{a}$ & Model $2^{b}$ \\
\hline$\geq 4$ High-quality embryos & $2.80(1.90-4.13),<0.001^{*}$ & $2.47(1.64-3.70),<0.001^{*}$ & $1.88(1.20-2.95), 0.006^{*}$ \\
\hline$\geq 35 \%$ High-quality embryos/ova captured & $0.97(0.69-1.37), 0.876$ & $0.94(0.65-1.34), 0.717$ & $1.05(0.69-1.58), 0.832$ \\
\hline$\geq 2$ blastocyst embryos & $3.40(2.33-4.95),<0.001^{*}$ & $3.54(2.36-5.33),<0.001^{*}$ & $3.30(2.11-5.17),<0.001^{*}$ \\
\hline$\geq 35 \%$ blastocyst embryos /ova captured & $1.96(1.31-2.92), 0.001^{*}$ & $2.01(1.30-3.10), 0.002^{*}$ & $1.94(1.18-3.18), 0.009^{*}$ \\
\hline Implantation (pregnancy) ${ }^{c}$ & $1.79(1.25-2.57), 0.002^{*}$ & $1.53(1.05-2.23), 0.028^{*}$ & $1.49(0.97-2.30) \cdot 0.070$ \\
\hline
\end{tabular}

Values are Odds ratio (95\% confidence interval), $p$-value. ${ }^{*}$ indicates a significant result $(p<0.05$, two-tailed)

Crude model $=$ odds ratios are no adjusted

${ }^{a}$ Model $1=$ odds ratios are adjusted by age and BMI. Note: 48 patients (41 implanted cycles) were missing BMI information

${ }^{\mathrm{b}}$ Model 2 =odds ratios are adjusted by age, BMI, and AFC

c Note: only 283 patients had their embryos transferred

on Day 2. Therefore, MOSI can be utilized as an additional and early measure to improve IVF outcomes, as the stimulation can be canceled, giving the patient a better chance with another stimulation protocol, potentially yielding a better outcome.

Here, using either OSI or MOSI, we identified 9.9\% of the IVF cycles as poor responders (data not shown). In poor responders, dehydroepiandrosterone (DHEA) promotes early follicular development [25], a higher number of oocytes at harvesting, and Day 3 embryos with improved clinical pregnancy rates as well as live birth rates $[26,27]$. DHEA, coupled with MOSI, could indicate if a previously identified poor responder will yield improved IVF parameters; however, future studies are required. Another concern for poor responders is the premature rise of LH. Even though gonadotropin-releasing hormone analogs can prevent the premature rise [28], the potential LH surge remains [29]. When follicle recruitment during the luteal phase is performed using luteal phase ovarian stimulation (LPOS) [30], LPOS did augment oocytes pick-up and the number of Day $3 \mathrm{em}$ bryos, but there was no difference in the pregnancy rate [31]. Again, LPOS, coupled with MOSI, could be another option for poor responders.

Table 4 Test efficiency of the Modified Ovarian Sensitivity Index for in vitro fertilization parameters (additional cohort)

\begin{tabular}{|c|c|c|c|c|c|c|c|c|c|}
\hline \multirow[t]{2}{*}{ Category } & \multirow{2}{*}{$\begin{array}{l}\text { MOSI } \\
\text { score }\end{array}$} & \multicolumn{4}{|c|}{ All data $(n=337)$} & \multicolumn{4}{|c|}{ Only normo-responders $(n=255)$} \\
\hline & & Prevalence & Test accuracy & PPV & NPV & Prevalence & Test accuracy & PPV & NPV \\
\hline \multirow[t]{6}{*}{ High-quality Embryo on Day 2 ( $\geq 6)$} & 2.1 & $27.3 \%$ & $33.2 \%$ & $28.6 \%$ & $88.5 \%$ & $27.1 \%$ & $27.1 \%$ & $27.1 \%$ & N/A \\
\hline & 2.6 & & $43.4 \%$ & $32.0 \%$ & $93.5 \%$ & & $35.7 \%$ & $29.4 \%$ & $95.8 \%$ \\
\hline & 3.1 & & $54.3 \%$ & $36.0 \%$ & $89.6 \%$ & & $50.2 \%$ & $33.7 \%$ & $88.3 \%$ \\
\hline & 3.6 & & $68.5 \%$ & $45.2 \%$ & $86.4 \%$ & & $69.0 \%$ & $45.1 \%$ & $85.0 \%$ \\
\hline & 4.1 & & $71.8 \%$ & $47.1 \%$ & $76.2 \%$ & & $73.3 \%$ & $57.1 \%$ & $73.8 \%$ \\
\hline & 4.6 & & $71.5 \%$ & $16.7 \%$ & $72.5 \%$ & & $72.9 \%$ & N/A & $72.9 \%$ \\
\hline \multirow[t]{6}{*}{ Blastocysts ( $\geq 6)$} & 2.0 & $35.9 \%$ & $39.5 \%$ & $36.9 \%$ & $80.0 \%$ & $35.7 \%$ & $35.7 \%$ & $35.7 \%$ & $\mathrm{~N} / \mathrm{A}$ \\
\hline & 2.5 & & $44.8 \%$ & $39.1 \%$ & $89.5 \%$ & & $35.7 \%$ & $35.7 \%$ & $\mathrm{~N} / \mathrm{A}$ \\
\hline & 3.0 & & $54.9 \%$ & $43.4 \%$ & $81.4 \%$ & & $49.0 \%$ & $39.8 \%$ & $76.6 \%$ \\
\hline & 3.5 & & $68.0 \%$ & $54.0 \%$ & $80.7 \%$ & & $66.3 \%$ & $52.1 \%$ & $78.3 \%$ \\
\hline & 4.0 & & $69.4 \%$ & $64.1 \%$ & $70.7 \%$ & & $68.2 \%$ & $75.0 \%$ & $67.7 \%$ \\
\hline & 4.5 & & 65.3 & $70.0 \%$ & $65.1 \%$ & & $64.3 \%$ & $\mathrm{~N} / \mathrm{A}$ & $64.3 \%$ \\
\hline \multirow[t]{7}{*}{ Rate of blastocyst/ova captured ( $\geq 35 \%$ ) } & 2.0 & $18.1 \%$ & $22.8 \%$ & $18.6 \%$ & $90.0 \%$ & $18.0 \%$ & $18.0 \%$ & $18.0 \%$ & $\mathrm{~N} / \mathrm{A}$ \\
\hline & 2.5 & & $28.2 \%$ & $19.7 \%$ & $94.7 \%$ & & $18.0 \%$ & $18.0 \%$ & N/A \\
\hline & 3.0 & & $43.0 \%$ & $22.1 \%$ & $91.2 \%$ & & $37.6 \%$ & $20.4 \%$ & $89.1 \%$ \\
\hline & 3.5 & & $61.4 \%$ & $28.6 \%$ & $91.5 \%$ & & $62.0 \%$ & $28.2 \%$ & $90.6 \%$ \\
\hline & 4.0 & & $74.8 \%$ & $31.3 \%$ & $85.0 \%$ & & $79.6 \%$ & $35.0 \%$ & $83.4 \%$ \\
\hline & 4.5 & & $80.7 \%$ & $30.0 \%$ & $82.3 \%$ & & $82.0 \%$ & $\mathrm{~N} / \mathrm{A}$ & $82.0 \%$ \\
\hline & 4.8 & & $81.3 \%$ & $0 \%$ & $81.8 \%$ & & $82.0 \%$ & N/A & $82.0 \%$ \\
\hline
\end{tabular}

Abbreviations: MOSI modified ovarian sensitivity index, NPV negative predictive value, and PPV Positive predictive value Bold values are the results based on the cutoff using the highest Youden index based on the Receiver-operating characteristic curve 
To reiterate, one of the key prognostic factors for IVF is collecting the optimal number of oocytes, 8 to 14 , and many researchers are developing indices to predict the optimal initial FSH dose to achieve this goal. For example, La Marca et al. developed a normogram, based on the patient's age as well as serum AMH and FSH levels that was to indicate the optimal initial FSH dose [32], and when compared to local protocols, it was determined that $\sim 13 \%$ of patients received a dose that was either suboptimal or excessive [33]. With high predictability, this index led to improve IVF parameters. MOSI would also complement this index, indicating if the initial dose was sufficient. However, women with PCOS remain under-evaluated and this is further confounded when considering the extreme variability of severity associated with PCOS and its associated comorbidities: insulin resistance, depression, hypertension, etc. [34]. Insulin resistance significantly augments cancellation rates and diminishes conception rates in PCOS women during IVF $[35,36]$. Therefore, any index to be used on PCOS women, such as MOSI, the La Marca normogram, or POSEIDON, should be evaluated for insulin resistance's effect on FSH stimulation. MOSI determines the effectiveness of the initial FSH dose; however, it could be inaccurate for severe insulin-resistant women, independent of PCOS. Another confounder would be the treatment with Metformin or any insulin-sensitizing agent. Metformin was shown to improve insulin resistance as well as the number of oocytes collected [37]; therefore, future studies would be required to determine the effect insulin resistance has on MOSI and other indices with PCOS or insulin-resistant women at predicting ovarian response and IVF outcomes.

This study has a few limitations. First, even though we represented the data using Generalized Additive Models, the associations were assessed with linear regression. The model would fit better using other possibilities; however, the majority of the graph appeared linear. Therefore, the association can be assessed over a limited range. Second, the cohort was made of women suffering from many different etiologies of infertility. It is possible that MOSI could be affected by the cause of infertility. Third, the subsequent FSH doses varied significantly from a similar initial dose. This would affect the associations; however, the change in subsequent FSH doses is dependent upon many factors.

\section{Conclusions}

Here, MOSI, an index based on the initial FSH dose and follicle development, allows the prediction of ovarian stimulation outputs and if the antagonist-based IVF cycle will lead to the development of high-quality embryos. This will allow a clinician to be able to decide to continue with the cycle or to change to a new stimulation in a subsequent cycle. The importance of this work lies in the high costs associated with reaching a follicular aspiration procedure with poor ova quality. Lastly, this study lays the groundwork for future prospective studies, in which MOSI could be used to modify FSH concentrations during an antagonist-based ovarian stimulation, promoting optimal ova production without leading to the development of ovarian hyperstimulation syndrome or other adverse effects.

\section{Acknowledgments}

We would like to thank the participants of the study, to Alexis Montes de Oca from LIDMOL for aiding in data collection, and to Dr. Leonardo M. Porchia for the drafting and critical analysis of the manuscript.

\section{Authors' contributions}

$\mathrm{DCM}, \mathrm{UG}$, and FCD made substantial contributions to the conception and design of the work; IM, GO, and FCD were the clinicians in charge; UG and DCM performed the acquisition and analysis of the data; ELB interpreted the data, drafted the original manuscript and revised it critically for intellectual content; ELB and FCD approved the final version of the manuscript to be published. All authors agree in all aspects of the work, ensuring that questions related to the accuracy or integrity of any part of the work were appropriately solved. The author(s) read and approved the final manuscript

Funding

None.

\section{Availability of data and materials}

The datasets used and analyzed for the current study are available from the corresponding author on reasonable request.

Ethics approval and consent to participate

Written informed consent was obtained, conducted in accordance with the Declaration of Helsinki. The Ethics Committee of the Ingenes Institute approved this study (approval number: ISF150218).

\section{Consent for publication}

Not applicable.

\section{Competing interests}

The authors declare that they have no competing interests.

\section{Author details}

${ }^{1}$ Clinical Research, Instituto de Infertilidad y Genética México SC, Ingenes, México City, Mexico. ${ }^{2}$ Departamento de Toxicología, Centro de Investigación y de Estudios Avanzados del Instituto Politécnico Nacional (CINVESTAV-IPN), Avenida Instituto Politécnico Nacional 2508, San Pedro Zacatenco, 07360 México City, Mexico.

Received: 17 April 2020 Accepted: 16 July 2020

Published online: 17 August 2020

\section{References}

1. Polis CB, Cox CM, Tunçalp Ö, McLain AC, Thoma ME. Estimating infertility prevalence in low-to-middle-income countries: an application of a current duration approach to demographic and health survey data. Hum Reprod. 2017;32(5):1064-74.

2. Myers ER, McCrory DC, Mills AA, Price TM, Swamy GK, Tantibhedhyangkul J, et al. Effectiveness of assisted reproductive technology (ART). UK: Centre for Reviews and Dissemination; 2008.

3. Pacchiarotti A, Selman H, Valeri C, Napoletano S, Sbracia M, Antonini G, et al. Ovarian stimulation protocol in IVF: an up-to-date review of the literature. Curr Pharm Biotechnol. 2016;17(4):303-15.

4. Tal R, Seifer DB. Ovarian reserve testing: a user's guide. Am J Obstet Gynecol. 2017;217(2):129-40.

5. Vermey BG, Chua SJ, Zafarmand MH, Wang R, Longobardi S, Cottell E, et al. Is there an association between oocyte number and embryo quality? A systematic review and meta-analysis. Reprod BioMed Online. 2019;1(39): 731-63. 
6. Sunkara SK, Rittenberg V, Raine-Fenning N, Bhattacharya S, Zamora J, Coomarasamy A. Association between the number of eggs and live birth in IVF treatment: an analysis of 400135 treatment cycles. Hum Reprod. 2011; 26(7):1768-74.

7. Rombauts L, Lambalk CB, Schultze-Mosgau A, van Kuijk J, Verweij P, Gates D, et al. Intercycle variability of the ovarian response in patients undergoing repeated stimulation with corifollitropin alfa in a gonadotropin-releasing hormone antagonist protocol. Fertil Steril. 2015;104(4):884-90. e2.

8. Biasoni V, Patriarca A, Dalmasso P, Bertagna A, Manieri C, Benedetto C, et al. Ovarian sensitivity index is strongly related to circulating $\mathrm{AMH}$ and may be used to predict ovarian response to exogenous gonadotropins in IVF. Reprod Biol Endocrinol. 2011;9(1):112.

9. Huber M, Hadziosmanovic N, Berglund L, Holte J. Using the ovarian sensitivity index to define poor, normal, and high response after controlled ovarian hyperstimulation in the long gonadotropin-releasing hormoneagonist protocol: suggestions for a new principle to solve an old problem. Fertil Steril. 2013;100(5):1270-6.

10. Abbara A, Patel A, Tia HJ, Clarke SA, Chia G, Eng PC, et al. FSH requirements for follicle growth during controlled ovarian stimulation. Journal. 2019. https://doi.org/10.3389/fendo.2019.00579.

11. The Society for Assisted Reproductive Technology (SART): The Society for Assisted Reproductive Technology; [updated 2019. Available from: https:// www.sart.org/. Accessed 1996-2020.

12. Başak Balaban DB, Calderón G, Catt J, Conaghan J, Cowan L, Munné S, Royere D, Van den Abbeel E. The Istanbul consensus workshop on embryo assessment: proceedings of an expert meeting. Hum Reprod. 2011;26(6): 1270-83.

13. Wackerly DMI, William / Scheaffer, Richard L. Estadistica Matematica Con Aplicaciones / 7 Ed. Learning C, editor 2010.

14. Hanley JA, Mcneil BJ. A method of comparing the areas under receiver operating characteristic curves derived from the same cases. Radiology. 1983;148(3):839-43.

15. Loh $S$, Wang JX, Matthews CD. The influence of body mass index, basal FSH and age on the response to gonadotrophin stimulation in non-polycystic ovarian syndrome patients. Hum Reprod. 2002;17(5):1207-11.

16. Oudshoorn S, Van Tilborg C, Hamdine O, Torrance H, Eijkemans M, Lentjes E, et al. Ovarian response to controlled ovarian hyperstimulation: what does serum FSH say? Hum Reprod. 2017;32(8):1701-9.

17. Capalbo A, Treff N, Cimadomo D, Tao X, Ferrero S, Vaiarelli A, et al. Abnormally fertilized oocytes can result in healthy live births: improved genetic technologies for preimplantation genetic testing can be used to rescue viable embryos in in vitro fertilization cycles. Fertil Steril. 2017;108(6): 1007-15.

18. Borges E Jr, Zanetti BF, Setti AS, Braga DP, Figueira RCS, laconelli A Jr. FSH dose to stimulate different patient'ages: when less is more. JBRA Assisted Reprod. 2017;21(4):336.

19. Pan W, Tu HT, Jin L, Hu C, Li YH, Wang RJ, et al. Decision analysis about the cost-effectiveness of different in vitro fertilization-embryo transfer protocol under considering governments, hospitals, and patient. Journal. 2019. https://doi.org/10.1097/MD.0000000000015492.

20. Hassan AMA, Kotb MMM, AwadAllah AMA, Shehata NAA, Wahba A. Follicular sensitivity index (FSI): a novel tool to predict clinical pregnancy rate in IVF/ICSI cycles. J Assist Reprod Genet. 2017;34(10):1317-24.

21. Bosch E, Labarta E, Kolibianakis E, Rosen M, Meldrum D. Regimen of ovarian stimulation affects oocyte and therefore embryo quality. Fertil Steril. 2016; 105(3):560-70.

22. Abbara A, Jayasena CN, Christopoulos G, Narayanaswamy S, Izzi-Engbeaya C, Nijher GM, et al. Efficacy of kisspeptin-54 to trigger oocyte maturation in women at high risk of ovarian hyperstimulation syndrome (OHSS) during in vitro fertilization (IVF) therapy. J Clin Endocrinol Metab. 2015;100(9):3322-31.

23. Thongphakdee A, Berg DK, Tharasanit T, Thongtip N, Tipkantha W, Punkong C, et al. The impact of ovarian stimulation protocol on oocyte quality, subsequent in vitro embryo development, and pregnancy after transfer to recipients in Eld's deer (Rucervus eldii thamin). Theriogenology. 2017;91:134-44.

24. Lin H-CA, Dutta R, Mandal S, Kind A, Schnieke A, Razansky D. Advancing ovarian folliculometry with selective plane illumination microscopy. Journal. 2016. https://doi.org/10.1038/srep38057.

25. Prizant $\mathrm{H}$, Gleicher $\mathrm{N}$, Sen $\mathrm{A}$. Androgen actions in the ovary: balance is key J Endocrinol. 2014;222(3):R141-51.

26. Chern CU, Tsui KH, Vitale SG, Chen SN, Wang PH, Cianci A, et al. Dehydroepiandrosterone (DHEA) supplementation improves in vitro fertilization outcomes of poor ovarian responders, especially in women with low serum concentration of DHEA-S: a retrospective cohort study. Reprod Biol Endocrinol. 2018;16(1):90.

27. Xu L, Hu C, Liu Q, Li Y. The effect of Dehydroepiandrosterone (DHEA) supplementation on IVF or ICSI: a meta-analysis of randomized controlled trials. Geburtshilfe Frauenheilkd. 2019;79(7):705-12.

28. Allegra A, Marino A, Coffaro F, Scaglione P, Sammartano F, Rizza G, et al. GnRH antagonist-induced inhibition of the premature LH surge increases pregnancy rates in IUI-stimulated cycles. A prospective randomized trial. Hum Reprod. 2007;22(1):101-8.

29. Reichman DE, Zakarin L, Chao K, Meyer L, Davis OK, Rosenwaks Z. Diminished ovarian reserve is the predominant risk factor for gonadotropin-releasing hormone antagonist failure resulting in breakthrough luteinizing hormone surges in in vitro fertilization cycles. Fertil Steril. 2014;102(1):99-102.

30. Zhang W, Wang M, Wang S, Bao H, Qu Q, Zhang N, et al. Luteal phase ovarian stimulation for poor ovarian responders. JBRA Assist Reprod. 2018; 22(3):193-8.

31. Lin LT, Vitale SG, Chen SN, Wen ZH, Tsai HW, Chern CU, et al. Luteal phase ovarian stimulation may improve oocyte retrieval and oocyte quality in poor ovarian responders undergoing in vitro fertilization: preliminary results from a single-center prospective pilot study. Adv Ther. 2018;35(6):847-56.

32. La Marca A, Papaleo E, Grisendi V, Argento C, Giulini S, Volpe A. Development of a nomogram based on markers of ovarian reserve for the individualisation of the follicle-stimulating hormone starting dose in in vitro fertilisation cycles. Bjog. 2012;119(10):1171-9.

33. Papaleo E, Zaffagnini S, Munaretto M, Vanni VS, Rebonato G, Grisendi V, et al. Clinical application of a nomogram based on age, serum FSH and AMH to select the FSH starting dose in IVF/ICSI cycles: a retrospective twocentres study. Eur J Obstet Gynecol Reprod Biol. 2016;207:94-9.

34. Sirmans SM, Parish RC, Blake S, Wang X. Epidemiology and comorbidities of polycystic ovary syndrome in an indigent population. J Investig Med. 2014; 62(6):868-74.

35. Fica S, Albu A, Constantin M, Dobri GA. Insulin resistance and fertility in polycystic ovary syndrome. J Med Life. 2008; (4):415-22.

36. Dale PO, Tanbo T, Haug E, Abyholm T. The impact of insulin resistance on the outcome of ovulation induction with low-dose follicle stimulating hormone in women with polycystic ovary syndrome. Hum Reprod. 1998; 13(3):567-70.

37. Fedorcsák P, Dale PO, Storeng R, Abyholm T, Tanbo T. The effect of metformin on ovarian stimulation and in vitro fertilization in insulin-resistant women with polycystic ovary syndrome: an open-label randomized crossover trial. Gynecol Endocrinol. 2003;17(3):207-14.

\section{Publisher's Note}

Springer Nature remains neutral with regard to jurisdictional claims in published maps and institutional affiliations.
Ready to submit your research? Choose BMC and benefit from:

- fast, convenient online submission

- thorough peer review by experienced researchers in your field

- rapid publication on acceptance

- support for research data, including large and complex data types

- gold Open Access which fosters wider collaboration and increased citations

- maximum visibility for your research: over $100 \mathrm{M}$ website views per year

At $\mathrm{BMC}$, research is always in progress.

Learn more biomedcentral.com/submissions 